\title{
Application of Inelastic Design Spectrum to Capacity-Demand-Diagram Methods
}

\author{
Anil K. Chopra and Rakesh K. Goel
}

\begin{abstract}
An improved capacity-demand-diagram method that uses the well-known constantductility design spectrum for the demand diagram is developed and illustrated by examples. This method estimates the deformation of inelastic SDF systems consistent with the selected inelastic design spectrum, while retaining the attraction of graphical implementation of the ATC-40 Nonlinear Static Procedure.
\end{abstract}

\section{Introduction}

The Nonlinear Static Procedure in ATC-40 (Applied Technology Council, 1996) and FEMA-274 (FEMA, 1997) documents is based on the capacity spectrum method (Freeman et al., 1975) which is used to determine the seismic deformation of an SDF system derived from the pushover curve. This method is based on the belief that the earthquake-induced deformation of an inelastic SDF system can be estimated satisfactorily by an iterative method requiring analysis of a sequence of equivalent linear SDF systems, thus avoiding the response history analysis (RHA) of the inelastic SDF system.

The principal objective of this investigation is to develop improved simplified analysis procedures, based on capacity and demand diagrams using the wellestablished inelastic response (or design) spectrum (e.g., Chopra, 1995; Section 7.10).

\section{Inelastic Design Spectrum}

The excitation is characterized by the elastic design spectrum of Fig. 1, constructed by the procedures of Newmark and Hall (1982), as described in Chopra (1995; Section 6.9), with $\ddot{u}_{g o}=1 \mathrm{~g}, \dot{u}_{g o}=122 \mathrm{~cm} / \mathrm{s}(48 \mathrm{in} . / \mathrm{sec})$, and $u_{g o}=91.4 \mathrm{~cm}$ (36 in.), and amplification factors of $\alpha_{A}=2.71, \alpha_{V}=2.30$, and $\alpha_{D}=2.01$ for the medianplus-one-standard-deviation spectrum for $5 \%$ damping. 
Definition. A constant-ductility spectrum for an elastoplastic hysteretic system is a plot of $A_{y}$ versus the initial elastic period $T_{n}$ for selected values of $\mu$. The pseudoacceleration $A_{y}$ is related to the yield strength $f_{y}$ by

$f_{y}=\frac{A_{y}}{g} w$

where $w$ is the weight of the system. The yield strength reduction factor is given by

$$
R_{y}=\frac{f_{o}}{f_{y}}=\frac{A}{A_{y}}
$$

where

$$
f_{o}=\left(\frac{A}{g}\right) w
$$

is the minimum yield strength required for the structure to remain elastic during the earthquake; $A$ is the pseudo-acceleration ordinate of the elastic design spectrum at $\left(T_{n}, \zeta\right)$.

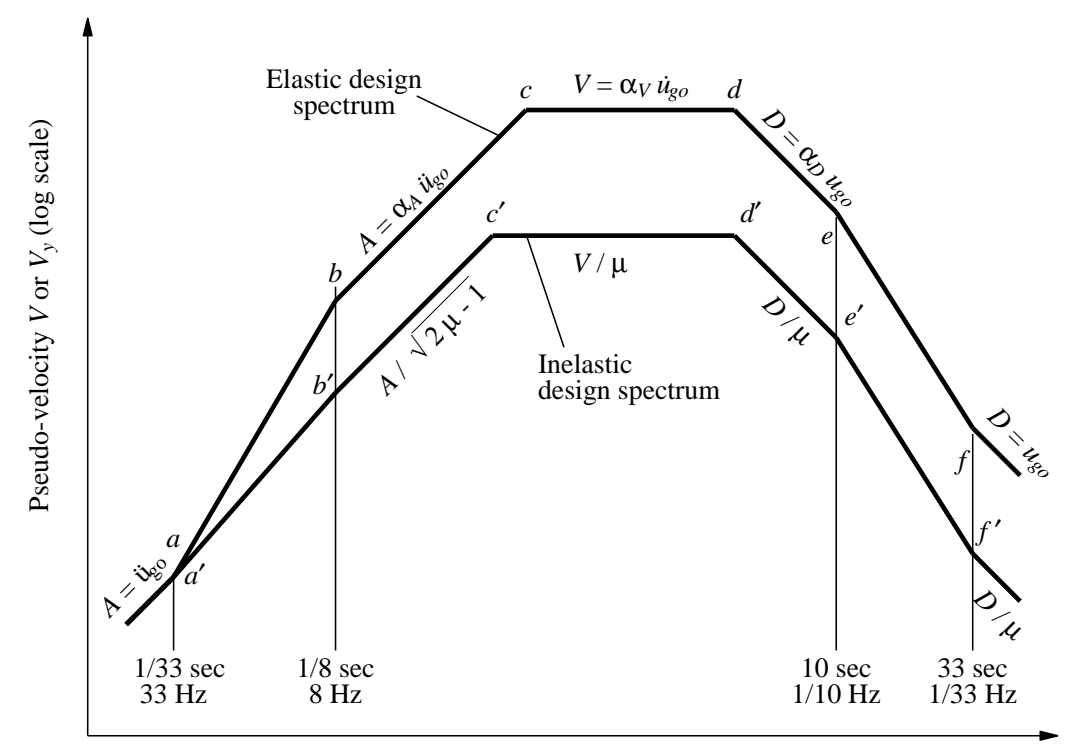

Natural vibration period $T_{n}$ ( $\log$ scale)

Figure 1. Construction of elastic and inelastic design spectrum.

A constant-ductility design spectrum is established by dividing the elastic design spectrum by appropriate ductility-dependent reduction factors that depend on $T_{n}$ (Fig. 1). The earliest recommendation for the reduction factor, $R_{y}$, goes back to the 
work of Veletsos and Newmark (1960), which is the basis for the inelastic design spectra developed by Newmark and Hall (1982).

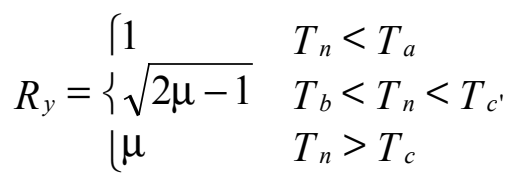

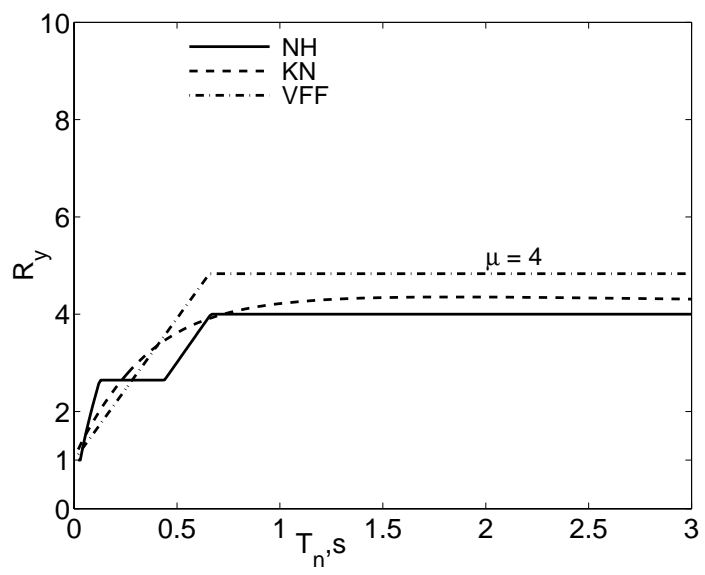

Figure 2. Variation of $R_{y}$ with $T_{n}$ for $\mu=4$ based on three different sources: Newmark and Hall (NH), Krawinkler and Nassar (KN), and Vidic, Fajfar, and Fischinger (VFF).

This equation is plotted in Fig. 2 for $\mu=4$. Starting with the elastic design spectrum of Fig. 1 and these $R_{y}-\mu$ relations for acceleration-, velocity-, and displacementsensitive spectral regions, the inelastic design spectrum constructed by the procedure described in Chopra (1995, Section 7.10), is shown in Fig. 3a.

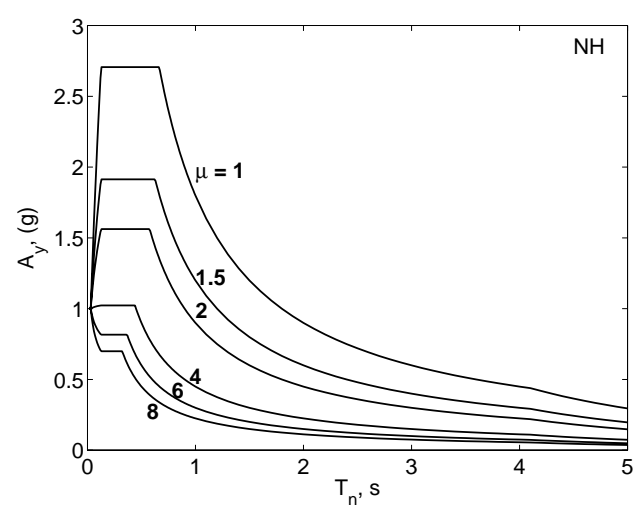

(a)

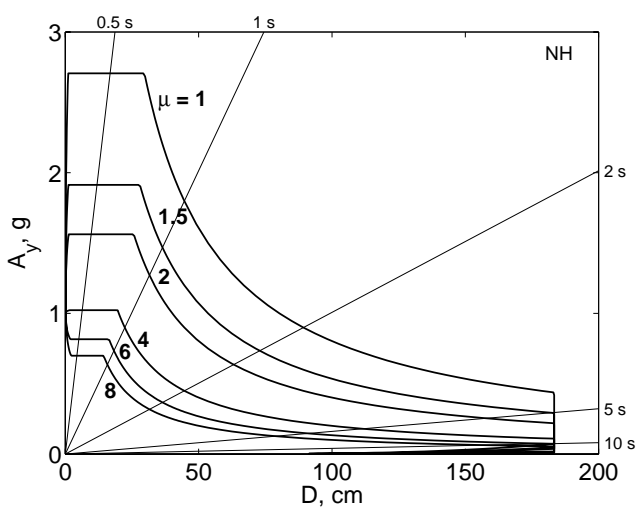

(b)

Figure 3. (a) Inelastic design spectra and (b) Inelastic demand diagrams, based on $\mathrm{NH}$ recommendations. 
In recent years, several recommendations for the reduction factor have been developed by regression analysis of the data from RHA of many SDF systems (e.g., Krawinkler and Nassar, 1992; Vidic, Fajfar, and Fischinger, 1994). Equations for these $R_{y}-\mu-T_{n}$ and resulting inelastic design spectra are available in Chopra and Goel (1999) and plotted in Fig. 2.

Yield Strength Demand from Design Spectrum. Given the design spectrum and the properties $T_{n}$ and $\zeta$ of an SDF system, we can readily determine the yield strength for the system consistent with a selected ductility factor $\mu$. Corresponding to $T_{n}, \zeta$ and $\mu$, the value of $A_{y} \div g$ is read from Fig. 3a. The desired yield strength is then given by Eq. (1).

Deformation Demand from Design Spectrum. An equation for the peak deformation can be derived in terms of $A_{y}$ as follows:

$u_{m}=\mu u_{y}$

with the yield deformation defined by

$$
u_{y}=\frac{f_{y}}{k}=\left(\frac{T_{n}}{2 \pi}\right)^{2} A_{y}
$$

where $k$ is the initial elastic stiffness of the system. Putting Eqs. (4) and (5) together gives

$$
u_{m}=\mu\left(\frac{T_{n}}{2 \pi}\right)^{2} A_{y}
$$

This equation can be expressed in terms of $A$, the pseudo-acceleration ordinate of the elastic design spectrum, by using Eq. (2)

$$
u_{m}=\mu \frac{1}{R_{y}}\left(\frac{T_{n}}{2 \pi}\right)^{2} A
$$

Presented in Fig. 4 are the deformations determined by using three different $R_{y}-\mu-T_{n}$ equations mentioned earlier. Observe that two of these recommendations lead to similar results except for $T_{n}<0.3 \mathrm{sec}$, indicating that the inelastic design spectrum is a reliable approach to estimate the earthquake-induced deformation of yielding systems, reliable in the sense that different researchers have produced similar results.

\section{Capacity-Demand-Diagram Method}

Demand Diagram. The inelastic design spectra of Fig. 3a will be plotted in the A-D format to obtain the corresponding demand diagrams. The peak deformation, $D=u_{m}$, of an inelastic system is determined from Eq. (8). Such data pairs $\left(A_{y}, D\right)$ are plotted to obtain the demand diagram for inelastic systems (Fig. 3b). 
Graphical Procedure. A procedure, which uses the demand diagram for inelastic systems (Fig. 3b), will be illustrated with reference to six elastoplastic systems defined by two values of $T_{n}=0.5$ and $1.0 \mathrm{sec}$ and three different yield strengths, given by Eq. (1) corresponding to $\mu=6,4$, and 2 (Systems 1 to 3), respectively. For systems with $T_{n}=$ $0.5 \mathrm{sec}, f_{y} \div w=0.5995,0.8992,1.5624$ and for $\mu=6,4$, and 2 , respectively. The corresponding values for systems with $T_{n}=1 \mathrm{sec}$ are $f_{y} \div w=0.2997,0.4496$, and 0.8992 (Systems 4 to 6). Superimposed on the demand diagrams are the capacity diagrams for three inelastic systems with $T_{n}=0.5 \mathrm{sec}$ (Fig. 5a) and $T_{n}=1.0 \mathrm{sec}$ (Fig. $5 b)$. The yielding branch of the capacity diagram intersects the demand diagram for several $\mu$ values. One of these intersection points, which remains to be determined, will provide the deformation demand. At the one relevant intersection point, the ductility factor calculated from the capacity diagram should match the ductility value associated with the intersecting demand curve. Determined according to this criterion, the deformation for each system is noted in Fig. 5. This result will be essentially identical to that given by Eq. (8). Implementation of this procedure is illustrated for two systems.

Examples. The yield deformation of System 1 is $u_{y}=3.724 \mathrm{~cm}$. The yielding branch of the capacity diagram intersects Newmark-Hall the demand curves for $\mu=1,2,4,6$, and 8 at $133.93 \mathrm{~cm}, 66.96 \mathrm{~cm}, 33.48 \mathrm{~cm}, 22.3 \mathrm{~cm}$, and $16.5 \mathrm{~cm}$, respectively (Fig. 5a). Dividing by $u_{y}$, the corresponding ductility factors are: $133.93 \div 3.724=35.96$ (which exceeds $\mu=1$ for this demand curve), $66.96 \div 3.724=17.98$ (which exceeds $\mu=2$ for this demand curve), $33.48 \div 3.724=8.99$ (which exceeds $\mu=4$ for this demand curve), $22.3 \div 3.724=6$ (which matches $\mu=6$ for this demand curve), and $16.5 \div 3.724=4.43$ (which is smaller than $\mu=8$ for this demand curve). Thus, the ductility demand is 6 and the deformation of System 1 is $\mathrm{D}=22.3 \mathrm{~cm}$.

For System 3, $u_{y}=9.681 \mathrm{~cm}$. The yielding branch of the capacity diagram intersects the Newmark-Hall demand curve for $\mu=1$ at $51.34 \mathrm{~cm}$ (Fig. 5a). The corresponding ductility factor is $51.34 \div 9.681=5.3$, which is larger than the $\mu=1$ for this demand curve. The yielding branch of the capacity diagram also intersects the demand curve for $\mu=2$ continuously from $9.681 \mathrm{~cm}$ to $25.2 \mathrm{~cm}$, which correspond to ductility factors of 1 to 2.6. The intersection point at $19.29 \mathrm{~cm}$ corresponds to ductility factor $=19.39 \div 9.681=2$ which matches $\mu=2$ for this demand curve. Thus, the ductility demand is 2 and the deformation of System 3 is $D=19.39 \mathrm{~cm}$.

Observe that for the presented examples, the ductility factor at the intersection point matched exactly the ductility value associated with one of the demand curves because the $f_{y}$ values were chosen consistent with the same $\mu$ values for which the demand curves have been plotted. In general this is not the case and interpolation between demand curves for two $\mu$ values would be necessary. Alternatively, the demand curves may be plotted at a finer $\mu$ interval avoiding the need for interpolation. 


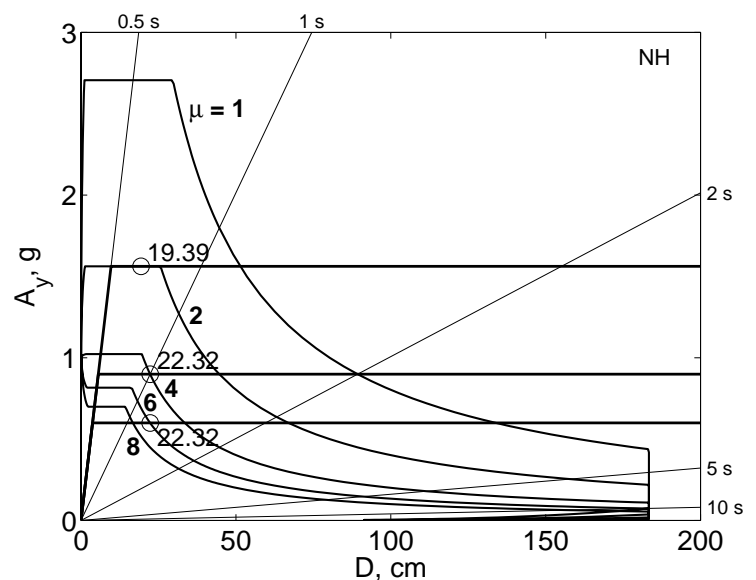

(a)

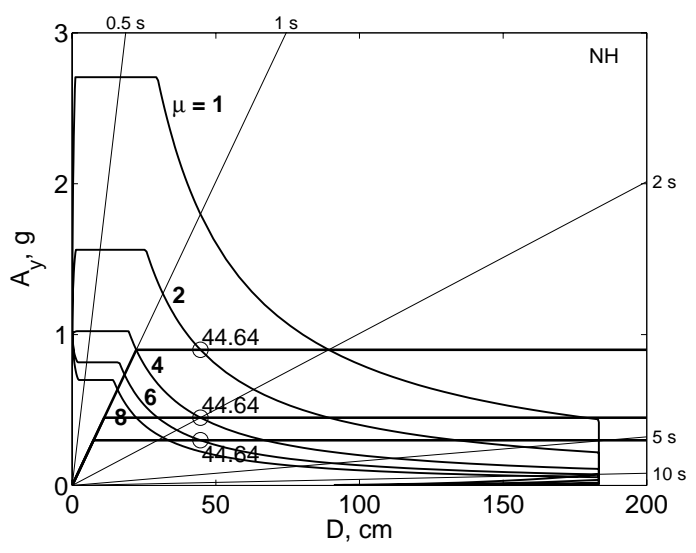

(b)

Figure 5. Application of Improved Procedure using NH inelastic design spectra: (a) Systems 1 to 3, and (b) Systems 4 to 6.

\section{Comparison with ATC-40 Method}

The deformation estimates by the ATC-40 method, as determined by Chopra and Goel (1999), are also included in Fig. 4. Relative to the deformation value from inelastic design spectra, the percentage discrepancy in the approximate result is plotted in Fig. 6. The ATC-40 method underestimates the deformation significantly, except for very long periods $\left(T_{n}>T_{f}\right.$ in Fig. 1). On the other hand, the proposed method gives deformations consistent with (exactly the same as) the selected inelastic design spectrum.

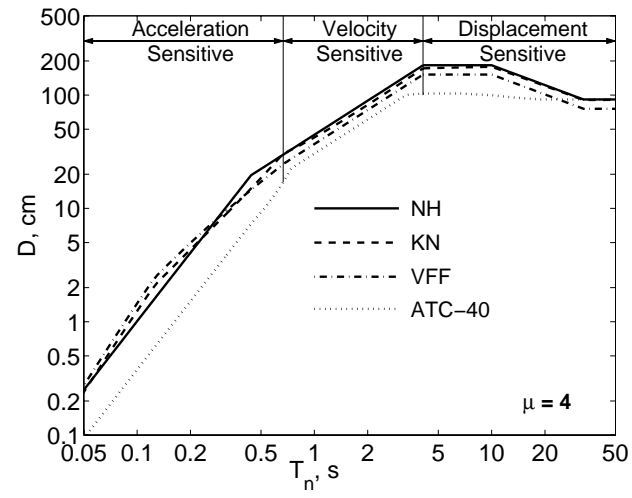

Figure 4. Deformation of inelastic systems $(\mu=4)$ determined from inelastic design spectra using ATC-40 Procedure and three (NH, KN, VFF) $R_{y}-\mu-T_{n}$ equations.

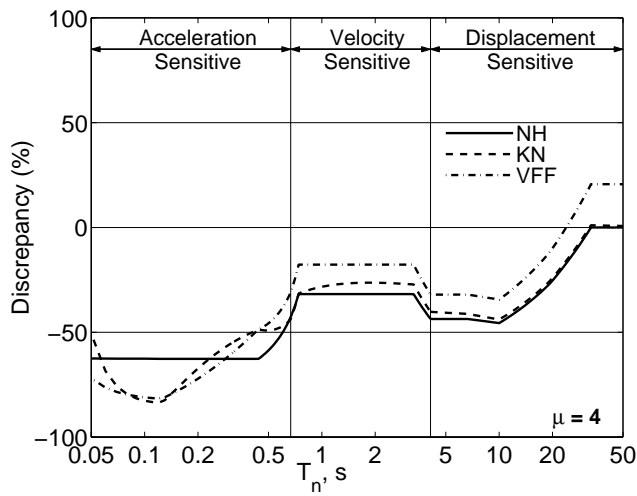

Figure 6. Discrepancy in deformations computed by ATC-40 procedure relative to three different (NH, KN, VFF) inelastic design spectra: $\mu=4$. 
In passing, note that the ATC-40 method is deficient relative to even the elastic design spectrum in the velocity-sensitive and displacement-sensitive regions $\left(T_{n}>T_{c}\right)$. For $T_{n}$ in these regions, the peak deformation of an inelastic system may be taken equal to that of the corresponding elastic system (Veletsos and Newmark, 1960; Chopra, 1995: Section 7.10), and the latter is given by the elastic design spectrum. However, the ATC40 procedure requires analyses of several equivalent linear systems and still produces worse results.

\section{Conclusions}

This investigation of capacity-demand-diagram methods to estimate the earthquakeinduced deformation of inelastic SDF systems has led to the following conclusions:

1. An improved capacity-demand-diagram method that uses the well-known constantductility design spectrum for the demand diagram has been developed and illustrated by examples. When both capacity and demand diagrams are plotted in the $A-D$ format, the yielding branch of the capacity diagram intersects the demand curves for several $\mu$ values. The deformation is given by the one intersection point where the ductility factor calculated from the capacity diagram matches the value associated with the intersecting demand curve.

2. The ATC-40 method significantly underestimates the deformation of inelastic systems for a wide range of $T_{n}$ and $\mu$ values compared to the value determined from the inelastic design spectrum using three different $R_{y}-\mu-T_{n}$ equations, all of which provided similar results.

In this paper we have focussed on improving the one step in the NSP or CSM to determine the earthquake induced deformation demand of an inelastic SDF system. In particular, the improved capacity-demand-diagram method presented here, while retaining the simplicity and graphical appeal of the NSP and CSM, provides results consistent with selected elastic design spectrum and chosen rules for constructing the inelastic design spectrum. Although illustrated for elastoplastic systems, this method is extendable to any force-deformation relation. However, additional work is necessary to evaluate approximations inherent in other steps of the NSP and CSM - in computing the pushover curve for a MDF system and converting it to a capacity diagram.

\section{Acknowledgement}

This research investigation is funded by the National Science Foundation under Grant CMS-9812531, a part of the U.S.-Japan Cooperative Research in Urban Earthquake Disaster Mitigation. This financial support is gratefully acknowledged.

\section{References}

Applied Technology Council (1996). Seismic Evaluation and Retrofit of Concrete Buildings. Report ATC 40, November. 
Chopra, A. K. (1995). Dynamics of Structures: Theory and Applications to Earthquake Engineering, Chapters 6, 7, and 13, Prentice Hall, New Jersey.

Chopra, A. K. and Goel, R. K. (1999). Capacity-Demand-Diagram Methods for Estimating Seismic Deformation of Inelastic Structures: SDF Systems. Report No. PEER1999/02, Pacific Earthquake Engineering Research Center, University of California, Berkeley, April.

FEMA (1997). NEHRP Guidelines for the Seismic Rehabilitation of Buildings, FEMA 273; and NEHRP Commentary on the Guidelines for the Seismic Rehabilitation of Buildings, FEMA 274, October, Federal Emergency Management Agency, Washington, D.C.

Freeman, S. A., Nicoletti, J. P., and Tyrell, J. V. (1975). "Evaluations of existing buildings for seismic risk - A case study of Puget Sound Naval Shipyard, Bremerton, Washington." Proceedings of $1^{\text {st }}$ U.S. National Conference on Earthquake Engineering, EERI, Berkeley, 113-122.

Krawinkler, H., and Nassar, A. A. (1992). "Seismic design based on ductility and cumulative damage demands and capacities." in Nonlinear Seismic Analysis and Design of Reinforced Concrete Buildings, P. Fajfar and H. Krawinkler, Eds., Elsevier Applied Science, New York, 1992.

Newmark, N. M., and Hall, W. J. (1982). Earthquake Spectra and Design. Earthquake Engineering Research Institute, Berkeley, CA.

Veletsos, A. S., and Newmark, N. M. (1960). "Effects of inelastic behavior on the response of simple system to earthquake motions." Proceedings of the $2^{\text {nd }}$ World Conference on Earthquake Engineering, Japan, Vol. 2, pp. 895-912.

Vidic, T., Fajfar, P., and Fischinger, M. (1994). "Consistent inelastic design spectra: strength and displacement." Earthquake Engineering and Structural Dynamics, Vol. 23, No. 5, pp. 507-521.

\section{Author Information}

Anil K. Chopra, Member of ASCE, Johnson Professor of Civil Engineering, Department of Civil and Environmental Engineering, University of California, Berkeley, CA 94720. Phone: (510) 642-1294. Fax: (510) 643-8928. Email: chopra@ce.berkeley.edu.

Rakesh K. Goel, Member of ASCE, Associate Professor, Department of Civil and Environmental Engineering, California Polytechnic State University, San Luis Obispo, CA 93407. Phone: (805) 756-2052. Fax: (805) 756-6330. Email: rgoel@calpoly.edu. 